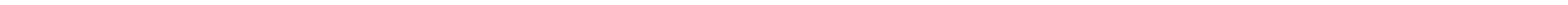




\section{Software for Bayesian Analysis : Current Status and Additional Needs}

by

Prem K. Goel

The Ohio State University

Columbus, Ohio 43210, U.S.A.

May 1987

Technical Report No. 366

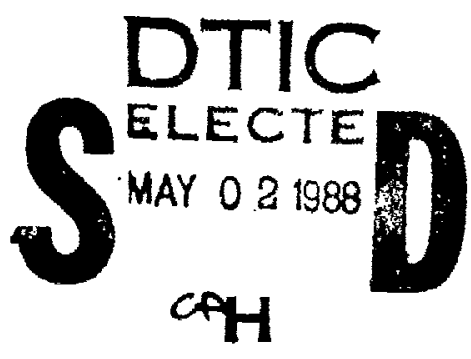

Prem K. Goel

The Ohio State University

Department of Statistics

Columbus, Ohio U.S.A. 
REPORT DOCUMENTATION PAGE

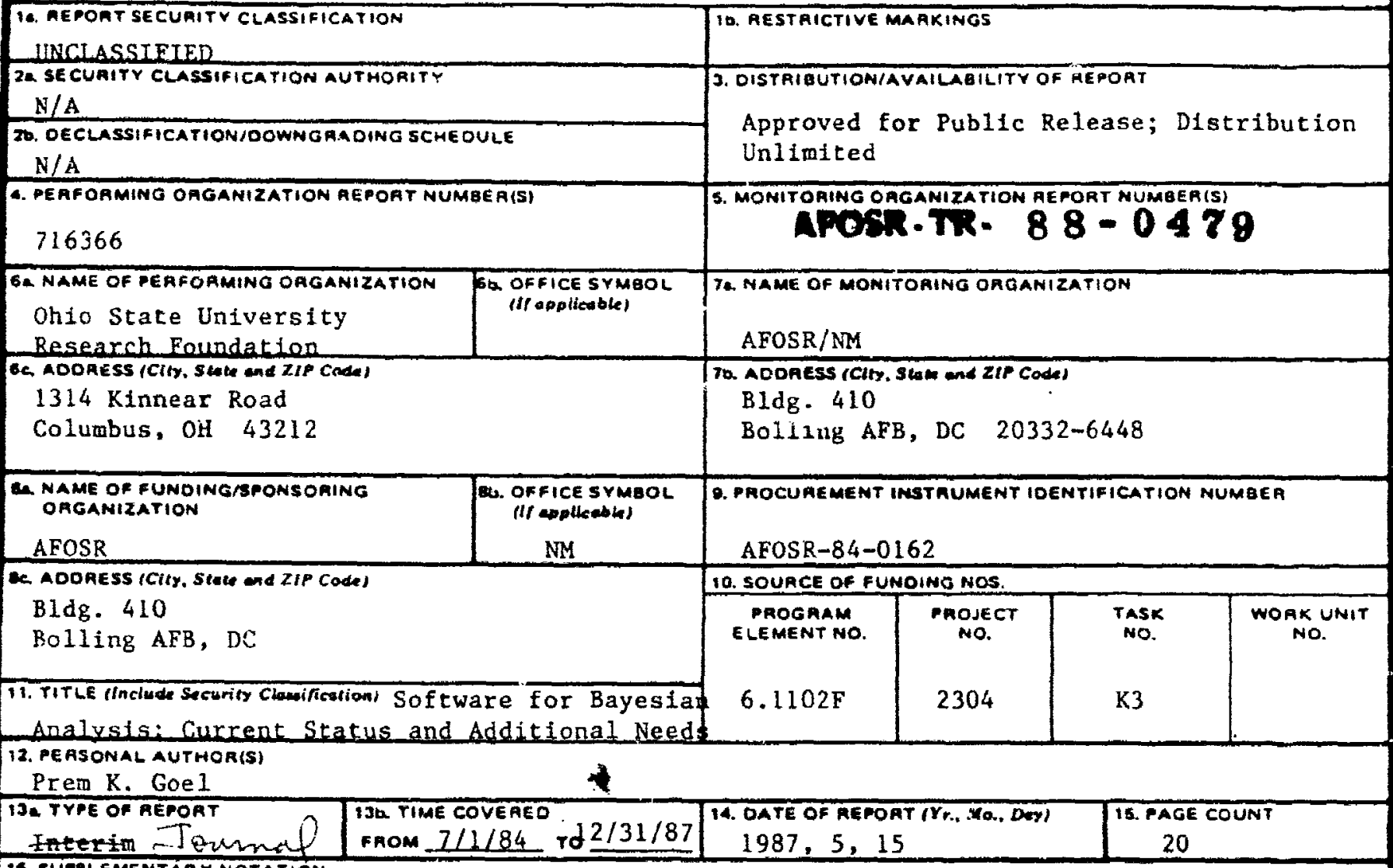
16. SUPPLEMENTAAT NOTATION

\begin{tabular}{|l|l|l|}
\hline 17. & \multicolumn{2}{|c|}{ COSATI coOES } \\
\hline EIELO & GAOUP & SUB. GA \\
\hline & & \\
\hline XXXXXXXXXXXXXXXXXXXXX \\
\hline
\end{tabular}

1a SUGJECT TEAMS (Centinue on Nowre 4 nacemary and identify by bloch nember)

Bayesian Software, Posterlor Distributions Summary

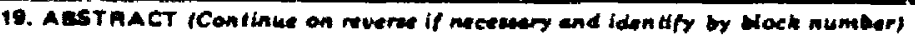

We make an attempt to provide comprehensive information about the existing software for data analysis within the Bayesian paradigm. The paucity of programs seems to indicate. that the Bayesian software available for widespread use is still in its infancy. We have a long way to go before a general purpose Bayesian 5tatistical Analysis Package is made available. Alternatives for reaching this goal quickly are presented in the concluding section.

\begin{tabular}{|c|c|c|}
\hline $\begin{array}{l}\text { 2Q OISTRIBUTIONIAVAILABILITY OF ABSTRACT } \\
\text { UNCLASSIFIEOIUNLIMITEO } \bigotimes \text { SAME AS RPT. } \square \text { OTIC USERS } \square\end{array}$ & $\begin{array}{l}\text { 21. ABSTRACT SECUAITY CLAC } \\
\text { UNCLASSIFIED }\end{array}$ & CATION \\
\hline $\begin{array}{l}\text { 22. Name of AEsponsibie Individual } \\
\text { Brian W. Woodroofe, Major USAF }\end{array}$ & $\begin{array}{l}\text { 22b. TELEPMONE NUMBEA } \\
\text { IInelude Ares Coda) } \\
\text { NM (202) 767-5025 }\end{array}$ & $\begin{array}{l}\text { 22C. OFFICE SYMBOL } \\
\text { AFOSR/NM }\end{array}$ \\
\hline
\end{tabular}




\title{
Software for Bayesian Analysis : Current Status and Additional Needs
}

\author{
ir $1 . ; j \quad$ Prem K. Goel \\ The Ohio State University \\ Columbus, Ohio 43210, U.S.A.
}

\section{Abstract}

We-make an attempt to provide comprehensive information about the existing software for data analysis within the Bayesian paradigm. The paucity of programs seems to indicate that the Bayesian software available for widespread use is still in its infancy. We have a long way to go before a general purpose Bayesian Staistical Analysis Package is made available. Alternatives for reaching this goal quickly are presented in the concluding section.

\section{Introduction.}

$\left.\therefore \quad T_{1}=5_{1} ;:\right\}$ $-\left\{c_{i}, \bar{s}\right.$

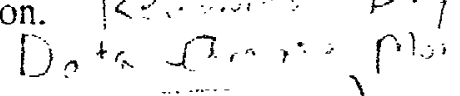

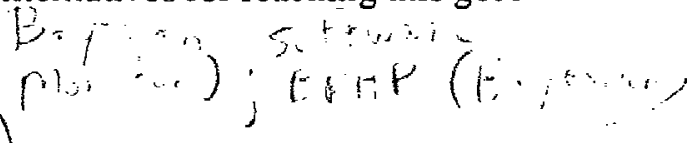

The starting point for this article was the workshop on Bayesian Statistical Compling, which was held at The Ohio State Universiiy during May 1986 and attended by approxinately 40 people involved in statistical computing, Bayesian decision analysis and AI. We organized this workshop to assess the status of Bayesian analysis software. We believe that Bayesian methodology will be used routinely by a widespread group of data analysts and scientists if a user friendly, general purpose Bayesian Statistical Analysis Package was available which could be used for class room teaching as well as for experimental data analysis. The workshop featured elcven talks concerning various issues in computational Bayesian analysis and some applications, as well as an open forum on Bayesian computing. The two main issues were (1) desirable computing environments for Bayesian statistical analysis, and (2) potentials for a Bayesian software package. There were diverse points of view about the environment suitable for an interactive Bayesian statistical analysis package, although all the participants seemed to believe that wide-spread use of Bayesian methodology will not become a reality without such a package. An overwhelming majority agreed with the notion that it is too early to push for a new statistical package. Instead, we should strive for new Bayesian software to be compatible with a package like ' $S^{\prime}$, in order to use its data handling and graphics capabilities. Furthermore, it was suggested that the Bayesian community should develop a 'Bajesian Bulletin Board', which will provide news about Bayesian programs as they are developed, and a 'Bayesian Software Databuse' which could be accessed for file transfers via popular computer networks. These task have not been
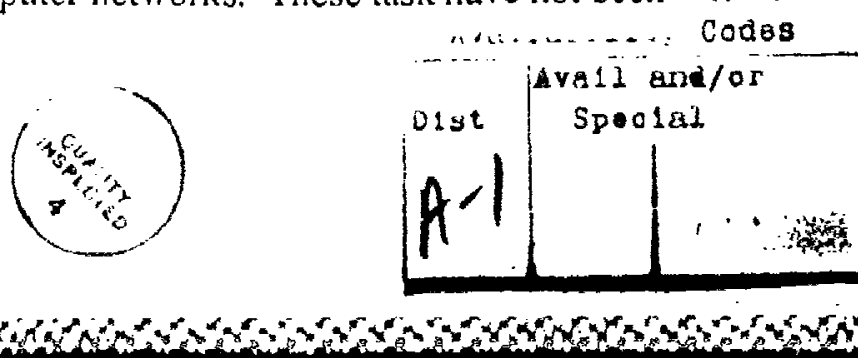
initiated in any meaningful way.

The information compiled in this article was provided by the individuals listed as contributors (within the parenthesis after the program name), in response to our survey questionnaire which was sent to approximately 450 Bayesian Statisticians and Econometricians in January 1987. An upda:ed SBIE mailing list along with some other lists were used to solicit this information. We have included all the responses. Thus the information is up-to-date as of April 1987. We believe that one large group of users of Bayesian methodology, namely engineers involved in risk assessment and reliability, have developed several special purpose programs which could be easily adapted for general reliability applications. However,we did not have access to any mailing list for this group, so the reliability programs listing can not be viewed as comprehensive.

One can view this article as an update of Press (1980) which listed Bayesian programs in existence at that time. If no updated information was recieved for a program listed in Press (1980), we have not included it in this paper with the belief that the program has either been superseded or else is no longer available. On comparing this paper with Press (1980), it is clear that impressive gains have been made in the developement of software for implementing Bayesian analysis paradigm for realistic specifications of prior information via approximations, numerical analysis, and Monte Cario integration. On the other hand, so few responses to our request for information indicates that only a few people have devoted their energy for developing general purpose Bayesian analysis software. We believe that this must change quickly if we want to see the 'Bayesian 21 st Century'.

The listing for programs in this paper follows the same general format as in Press (1980). The software has been listed according to the following categories:

(i) CADA, a general purpose data monitor (Section 2) (ii) Regression modeling, Econometric modeling and Time Series methodology (Section 3) (iii) Computation/Approximation of Posterior distribution, moments, quantiles and mode (Section 4) (iv) Elicitation of prior information (Section 5) (v) Rriliability Analysis (Section 6) and (vi) Miscelleneous (Section 7).

In Section 8, we present our views on a quick development of a general purpose Bayesian Analysis Software Package.

2. CADA, a general purpose data analysis monitor.

Program Name: CADA [Computer Assisted Data Analysis Monitor(CADA Group)]

Function:

This monitor provides a conversational language for Bayesian analysis. It has gone through several updatings. The most recent version (1983) includes susbtintial enhancements over the (1980) version. The CADA operation has been moved to a private corporation now. CADA is a 
hierarchacally structured system with several component groups namely: Data Management facility, Simple Parametric models, Decision theoretic models, Full-rank ANOVA models, Sinultaneous estimation, Full-rank MANOVA, Exploratory Data Analysis, Psychometric methods, Probability distribution functions, and Actuarial functions.

Input:

Output:

Raw data to be entered in, on-line or data files to be loaded.

Analysis for Beta, two-parameter normal, and multinomial models using conjugate priors; assessment of conjugate priors and utility functions; full rank Model I ANOVA for and MANOVA for multifactor designs using conjugate for noninformative priors; elementary classical statistics; graphical evaluatuions of various probability distributions; multiple linear regression analysis and simultaneous estimation of regression in $\mathrm{m}$-groups.

Programing language: BASIC (Compiler or interpreter required on the machine) Machines: DEC-PDP-11(RSTS); DEC-VAX-11(VMS), PRIME, HP-3000. IBM PC version coming sool:.

Documentation: $\quad$ Novick, M.L., Hamer, R.M., Libby, D.L., Chen, J. J. and Woodworth, G. G.(1983), Manual for the Computer-Assisted Data Anaslysis (CADA) Monitor (1983), Iowa City, IA: The CADA Group, Inc..

Availability: $\quad$ The CADA Group, Inc., 306 Mullin Ave., Iowa City, IA 52240, Tel. \# (31D) $351-7200$

Remarks: $\quad$ (i) The CADA system was developed at The lowa State University under the direction of Late Prof. Melvin R. Novick.

(ii) The software is fully supported and is available at a cost of $\$ 600$ per copy.

3. Normal Linear Regression, Econometric models and Time Series Analysis.

Program Name: BRAP [Bayesian Regression Analysis Program(Abowd/Zellner)], Version 2.0

Function: This program provides a unitied package for the Bayesian analyses of the nomal linear multiple regression model (MRM) with multivariate normal erorrs under a noninformative prior, a g-prior or a natural conjugate prior distribution. Some numerical integration capability via Simpson's rule and Monte Carlo importance sampling also provides a facility for analysis of nonslandard models. Borh the prior and the posterior distributions of the regression coefficients can be analyzed. Plotting of raw data and residuals 
Input:

Output:

Programing language:

Machine:

Documentation:

Availability:

Remarks:

Program Name:

Function:

Programing language:

Machines:

Availability: and prior and posterior marginals and bivariate contours for regression coefficients can also be done. The pos'erior distribution of linear functions of regression coefficients, the realized error terms, and predictive distribution of the dependent variables can also be obtained. Some transfummations are already available and $\mathrm{IMSL}^{(1)}$ could be loaded for more transformations. The control cards are in JCL format. Data Files can be easily loaded using a load command.

Updates the prior parameters and plots Marginal and bivariate contours of the prior and the posterior distributions of the regression coefficients; posterior distribution of the realized errors; posterior distribution of linear functions of coefficients; sta. dard posterior information; quantiles of posterior distribution can also be obtained via numerical integration routines.

\section{FORTRAN-IV}

IBM-MVS (may need some modifications for newer IBM compilers)

Abowd, J.M., Moulton, B. R. and Zellner, A.(1985) The Bayesian

Regression Analysis Package, BRAP user's Manual Version 2.0 of Dec.

1985., H.G. B. Alexander Research Foundation, Graduate Schood Of

Business, University of Chicago

The package is available from Prof. Arnold Zellner, University of Chicago, Graduate School of Business at a very nominal cost.

(i) A new version may be coming soon according to Prof. Zellner.

(ii) Other contributers to the developement of BRAP include F. Finnegan, $S$. Grossman, C. Plosser, P. Rossi, A. Siow, J. Stafford, and W. Vandaele.

\section{BAP [Bayesian Analysis Package(de Alba/ Rocha)]}

This main program anbd subroutine package is an enhancement of BRAP in that it includes BRAP as well as subroutines for Bayesian Disaggregation and Constrained Forecasting.

FORTRAN 77

IBM PC and PC compatibles.

It is available from from Prof. Enrique de Alba, Instituto Technologico Autonomo De Mexico (ITAM), Rio Hondo, No. 1, Mexico, D.F. 01000 at a nominal cost of a diskette and miling charges. 
Program Name: SEARCH [Seeks Extreme and Average Regression Coefficient Hypothesis (Leamer/Leonard)]

Function:

This is a user-oriented package for Bayesian inference and sensitivity analysis that pools prior beliefs about the regression coefficients with evidence embodied in a given data set. Prior beliefs are assumed to be equivalent to a previous, but possibly fictitious data set. SEARCH offers a study of the sensitivity of the posterior estimates to changes in features of the prior beliefs expressed in terms of a fictitious data set.

Input: $\quad$ Formatted or free-format Card-Image files or on-line CRT input. Input files

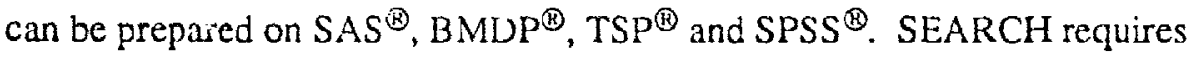
access to a double precision version of IMSL ${ }^{*}$ library.

Output: Diagnostic messages for debugging syntax errors are available. The program reports the summary of prior and data information received and computes the approximate posterior mode for the regression coefficients when the prior beliefs are modeled as if $\mathbf{R} \beta$ came from a nomal population with a specified mean $r$ and a covariar e matrix $V$. It also reports the sensitivity of the modal estimate to changes in the prior location $r$ and the prior covariance matrix $V$ in the form of extreme bounds for any linear function of the parameters specified by the user.

Programing language: FORTRAN IV. The manual for Version 6 states that it is not completely available in FORTRAN source code. Several of the subroutines for performing high precision arithmetic, that SEARCH calls for, are object code modules (written in IBM 370 machine code) and bulk of the SEARCH is written in FORTRAN IV that is compiled at UCLA on the IBM FORTRAN IV GI Compiler; i.e., not necessarily ANSI standard FORTRAN IV.

Machine: IBM $370 / 3033$.

Documentation: $\quad$ Leamer, E.E. and Leonard, H. B. (1985) User's Mamual for SEARCH-A sofnware package for Bayesian inference and sensitivity analysis, SERACH Version 6,Oct. 1985.

Availability: The program is available from Prof. E. E. Leamer, Department of Economics, UCLA, 405 Hilgurd Av., Los Angeles, CA 90024, (213) 825-1011, at $\$ 100$ per copy on an IBNI OS standard label 9 track 1600 BPI tape containing four card-image files. Cards or a 6250 BPI tape can be made available on special request. 
Remarks:

(i) The program was developed by Edward E. Leamer and Herman B.

Leonard. This version was programmed by Arvin Stidick and the MANUAL was extensively edited and largely rewritten by Thomas $E$. Wolff.

(ii) The Version 6 differs from Version 5 in its efficiency of computation and economy of input and output.

(iii) A latest example of how SEARCH can be used is given in Leamer, E. E. and Leonard, H.B.(1983) Reporting the fragility of Regression Estimates, The Review of Economics and Statistics.

Program Name: MICRO EBA [ Micro computer version of SEARCH(Fowles)]

Function:

This main program is the micro computer version of Leamer and Leonard's

Program SEARCH described above.

Programing language: GAUSS

Machine:

Any personal computer running GAUSS software package Version 1.46 or higher.

Availability: The program is available free of charge from Prof. Richard Fowles, Department of Economics, Rutgers University, Newark, NJ 07102.

Program Name: $\quad$ BRP [ Bayesian Regression Program (Bauwens)]

Function:

The main Program executes computations necessary for Bayesian regression analysis for various standard econometric models, discussed in Dreze(1977). The prior beliefs are modeled as Poly $-t$ densities.

Input:

Output:

Raw data as card-image files. Input data are echoed as output.

Posterior parameters and marginals of regression coefficients and precision and standard deviations; classical regression analysis, posterior residuals and predictive density function of the dependent variable; conditional posterior with given precision, conditional posteriors of some regression coefficients given some others, marginalized over the precision.

Programing language: FORTRAN 77

Machine: $\quad$ IBM $370 / 158$ at the University of Louvain. It is portable according to Dr. Bauwens. In near term, a $\mathrm{PC}$ version is possible.

Documentation: Bauwens, L. and Tompa, H. (1977) Bayestan Regression Program(BRP), CORE User's Manual Set \# A-5, and Tompa. H.(1977) Poly-t Distributions (PTD), CORE User's Manual Set \# C-9. 
Availability:

Remarks:

Program Name:

Function:

Input:

Output:

Programing language:

Machine:

Documentation:

Availability:

Program Name:

Function:

Output:
It is available from Prof. Luc Bauwens, CORE, 34 Voie Du Roman Paays, B-1348 Louvain-La-Neuve, Belgium at a cost of 5.000 Belgium Francs.

(i) BRP calls another program PTD to evaluate poly-t densities.

(ii) These programs have been developed by $H$. Tompa under the guidance of Profs. Jacques Dreze and Jean-Francois Richard and with assistance from Luc Bauwens, Jean-Paul Bulteau and Philippe Gille.

Fully Bayesian Analysis of ARMA Time Series Models(Monahan)

A collection of main program and subroutines carries out the Bayesian Analysis for ARMA time series models using natural conjugate priors as described in Monahan(1983).

Information not yet available

Programs compute the posterior and predictive distribution of paramaters for a given set of ARMA models using the natural conjugate prior. The graphical displays can be obtained via SAS/GRAPH.

FORTRAN 66

Portable

Monahan, J.(1980) 'A Structured Bayesian Approach to ARMA time series models, I,II,II', Technical Reports, Department of Statistics, North Carolina State University, Raleigh, NC.

The programs package is available on tape from Prof. John Monahan, Department of Statistics, North Carolina State University, P.O. Box 8203 , Raleigh, NC 27695 at a nominal charge.

\section{Sampling the Future (Thompson)}

This program simulates the predictive distribution of a set of future observations via Monte Carlo methods as discussed in Thompson (1986). The main program and several subroutines provide a Monte Carlo histogram for the predictive distribution of a future observation or a scattergram of samples from the predictive distribution of a pair of future observations. The model may contain as many as 10 ARMA parameters in upto 3 AR factors and upto 3 MA factors. Thus multiplicative seasonal factors and the difference factors may be used in the model. Estimation step allows either a diffuse or a conjugate nomal/ gamma Prior distribution. 
Programing language: FORTRAN 77 ANSI standard.

Machine: $\quad$ The program should run on any machine with standard FORTRAN 77

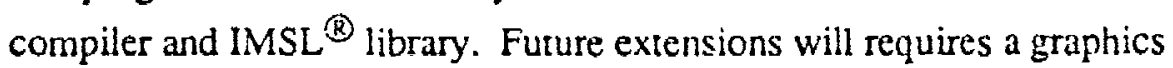
temina'. The program will run on a PC with a math cc-processor, but an AT type machine with a hard disk is recommended for realistic usage.

Availability: The package is available on a diskette, for a nominal charge of $\$ 10$, from Prof. Patrick Thompson, Faculty of Management Sciences, The Ohio State University, 1775 S. College Road, Columbus OH 43210.

Remarks:

(i) Future plans include a graphic display of predictive distributions and to add the algorithm for prediction from a set of ARMA models given in Monahan (1983).

Program Name: Bayes \& Empirical Bayes Shrinkage Estimation of Regression Coefficients (Nebebe)

Function:

The program computes Bayes and empirical Bayes Estimates for a multiple normal linear regression model in which the prior for the regression coefficients and the precision is modeled as a hierarchical normal with mean $\mu$ and precision $\tau^{2}$ and the hyperparameters are assumed to have various diffuse distributions, see Nebebe, F. and Stroud, T.W. F.(1986).

Programing language: FORTRAN, requires access to NAG library.

Documentation: No seperate documentation is available. The details are given in Nebebe, F. (1984) Ph. D. thesis, Deparment of Mathematics and Statistics, Queen's University, Kington, Canada.

Availability: $\quad$ The program is available from Prof. F. Nebebe, Dept. or Decision Sciences and MIS,Concordia University, 1455 De Maisonnevue Blvd. West, Montreal, Quebec H3G1M8, Canada

Remarks: $\quad$ (i) This program provides no capability which is not available in BRAP, SEARCH or BAP. But it may be useful for individuals who are interested in giving a workout to their NAG package.

Program Name: SHAZAM [General Econometrics program (White)]

Function:

The program provides a portable FORTRAN? program for general econometric modeling on a $P C$ for $\$ 250$ or a main frame for $\$ 500-900$. The 
Availability:

Program Name:

Function:

Output:

Programing language:

Documentation:

Availability:

Program Name:

Function:

Programing language:

Availability:

Program Name:

Function: author promises that the next version will include a Bayesian Iitequaliv regression and has not provided any other information.

Available from Prof. Kenneth J. White, Economics Deparment, L.... . . sity of British Columbia, Vancouver, B.C. Canada in case you are curious enoughi about the name.

BTS [Baycsian Time series (Carlin/Demuster)]

The program package carries out computations for Bayesian estimation of unobserved components('seasonal'/nonseasonal') in monthly time series under a class of Gaussian Mixed models as described in Carlin, Dempster and Jonas(1985). It uses likelihood based methods for estimation of model parameters.

The program provides posterior estimates of model parameters. A nonportable version for the A pollo DN600 workstation has many graphical capabilities.

\section{FORTRAN 77 (Standard ANSI)}

Description of the program is available in Carlin, J. B.(1987) Ph.D. Thesis, Department of Statistics, Harvard University

Available free of charge from Prof. A.P. Dempster, Department of Statistics, Harvard University, Science Center, 1 Oxford Street, Cambridge, MA 02138.

PROC SEQ [Sequential Scoring Algorithm(Blattenberger)]

The function performs iterative computation of forecasting distribution for the dependent variable of a normal linear model with a normal-gamma prior distribution or optional g-priors. Scores for five different scoring rules are also computed.

STAT80 Procedure, currently being converted to SAS ${ }^{\circledR}$ PROC MATRIX. Available free of charge from Prof. Gail Blattenberger, Deparment of Economics, University of Utah, Salt Lake City, UT.

MAXENT [ Data Analysis by Maximum Entropy Principle Version 1.17 (Jaynes)]

This beta test version of MAXENT provides fitting of an incompletely specified linear model of the form $Y=X F$, whe e the data vector is $Y$, the 
'smearing matrix' $\mathrm{X}$ is known but does not have full rank and the elements of the vector $F$ are non-negative and add to 1. The Maximum Entropy Principle, see Jaynes(1983) finds the solution to the above equation which maximizes $t$ he entropy of the probability distribution $\mathbf{F}$.

Input: The program is interactive. One needs to decide the accarcy level for satisfying all the constraints.

Output: The optimal solution is obtained in an iterative mode. The output for each iteraration can be printed.

Programing language: BASIC

Machines:

IBM PC and compatibles. An ASCII source code file is also on the diskette for transporting the program to other micro computers.

Documentation: Help fie and Manual on diskette.

Availability: Available free of charge from Prof. Ed T. Jaynes, Department of Physics, Washington University, St. Louis, MO 63130. Individuals sending comments and user experience to Prof. Jaynes will receive the Version 2.0 free.

The programs briefly discussed below have been written for applicaticrs of linear models to specific problems.

Program Name: RECONDA (Braithwait, Steven)

Function: $\quad$ This proyram incorporates engineering prior estimates of appliance level electricity consumption into a statistical analysis of household hourly consumption via a hierarchical linear model. The modeling details are given in Caves, Herriges, Train, Windle(1987).

Programing language: $\quad \mathrm{C}$

Machines:

IBM PC and PC compatibles

Availability:

The program will be distributed free of charge by EPRI, P.O. Box 10412 , Palo Alto, CA 94303 to EPRI member utilities, government and academic institutions.

Program Name: Statistical Cost Allocation (Wright, Roger)

Function:

Implements the indirect cost allocation methodolosy based on a multiple linear model as described in Wright(1983).

Prugraming language: FORTRAN 77 (Standard ANSI) 
Documentation: The program description and listing are given in Wright, R. and Oberg, $K$. (1983), The 1979-80 University of Michigan Heating Plant and Utilities Cost Allocation Study, Working Pazper \#352, Graduate School of Business Administration, The University of Michigan.

Availability: Available free of charge from Prof. Roger Wright, Graduate School of Business Administration, The University of Michigan, Ann Arbor, MI 48109.

\section{Computation/Approximation of Posterior Distribution Features.}

Program Names: Function:

Input:

Output:

Programing language: Machines:

Documentation:

Availability:

\section{Bayes Four and $g r$ (Smith, A.F. M.)}

The Bayes four system consists of a library of subroutines which is primarily intended for numerical computation of multiple integrals in interactive mode. The evaluation of the posterior distribution's features for a practical implementation of the Bayesian paradigm for up to 6 parameters using numerical integration procedures and upto 20 parameters using Monte Carlo integration. The $g r$ library consists of subroutines for an interactive color graphics system which can be used to reconstruct and display outpic of the BAYES FOUR system, for reference, see Smith, Skene, Shaw, Naylor, and Dransfield(1985).

Solving an inference problem requires writing additional program code for specific problem which can call BAYES FOUR and $g r$ subroutines.

The posterior moments and marginals can be evaluated by calling these menu driven subroutines. The $g r$ package can be used to provide graphical displays of the univariate and bivariate marginal posterior densities and predictive densities from outputs of BAYES FOUR. Bayes Four in FORTRAN 77; gr in 68000 assembler, C aind FORTRAN 77. BAYES FOUR is portable. However $g r$ has not been configured for any standard graphics system or workstation yet.

Naylor, J. C. and Shaw, J. E. H.(1985) BAYES FOUR-User Guide ; Naylor, J. C. and Shaw, J. E. H.(1985) BAYES F DUR-Implementation Guide; Shaw, J. E. H. (1985) gr User Guide. All these are technical reponts from the Nottingham Statistics Group, Department of Mathematics; University of Nottingham .

These systems may be made available by Prof. Adrian Smith, Department of 
Remarks:

Program Name:

Function:

Programing language:

Documentation:

Availability:

Remarks:

Program Name:

Function:
Mathematics, University of Nottingham, Nottingham, U.K.

(i) Th $;$ Nottingham Statistics Group is actively involved in developing numerical integration systems for implementing Bayesian me:hodology. Therefore some enhanced versions of these subroutine packages may be available soon.

(ii) For usage experience of this system on some interesting applied problems in pharmaceutical industry, see Racine, Grieve, Fluhler, and Smith (1986).

Simple Importance Sampling[Computation of Posterior moments and densities via Monte Carlo Integration (van Dijk)]

The program approximates multiple integrals that arise in the posterior moments and marginal densities of parameters of interest in econometric and statistical modeling, via a Monte Carlo integration method known as importance sampling.

\section{FORTRAN 77}

The algorithm, the program listing and some examples are given in van Dijk, H. K., Hop, J. P. and Louter, A. S.(1986) An algorithm for the computation of Posterior moments and densities using simple importance sampling. Econometric Institute Report 8625/A, Erasmus University, Rotterdam. The program is available from Prof. Herman K. Van Dijk, Econometric Institute, Erasmus University Rotterdam, P.O. Box 1738-3000 Dr. Rotterdam, The Netherlands.

(i) Some standard programs for the method of mixed integration [see, van Dijk, Kloek and Boender(1985)] are under preparation by Prof. van Dijk. (ii) Geweke, J.(1987) provides some interesting methods for constructing Importance Sampling density which are more flexible than the multivariate Student-t density. He has also developed a PC-AT version of Monte Carlo integration program which uses these more flexible methods. It is available on diskettes from Prof. John Geweke, Institute of Statistics and Decision Sciences, Duke University, Durham, NC 27706.

BAYES3/3D[ Multiparameter Univariate Bayesian Analysis using Monte Carlo Integration(Stewart)]

Bayesian inference for univariate response variable using Monte Carlo 
integration. Up to nine parameter flexibility allowed. Can handle usual random sampling data, interval data, censored data, binomial data at different stresses or times.

Input: $\quad$ Data and control cards as card-image files.

Output: $\quad$ Displays posterior means and posterior percentile curves for CDFs, hazard rate functions, or probability of failure(response) versus stress (dose) or time. (References: Stewart, L. $(1979,83,85)$.

Programing language: FORTRAN 77

Machines:

A graphics terminal is highly desireable but not absolutely necessary. Need DISPLA graphics software. GKS and DI-3000 versions are being written.

Documentation: $\quad$ Stewart, L. (1987) User's Manual for BAYES3/3D, A program for multiparameter univariate Bayesian analysis using Monte Carlo integration. Availability: The program was developed under various Federal contracts at Lockheed Palo Alto Research Laboratory, Palo Alto CA 94304. Dr. Leland Stewart, will provide the tape in individual cases, if he can get permission from Lockheed.

\section{Program Name: LINDLEY.BAS (Sloan)}

Function:

This BASIC subroutine performs algebraic manipulation and constructs the expanded formula for use of approximating the ratio of two integrals, required in the evaluations of the posterior distribution's features, as discussed in Lindley(1980).

Input: $\quad$ The program prompts for the number of parameters to be estimated.

Output: The printout gives the complete algebraic equation to needed to approximate the ratio of integrals.

Programing language: MS BASIC

Machine:

IBM PC or compatibles. Special printing customized for EPSON series of printers.

Availability: $\quad$ Available free of charge from Prof. Jeff A. Sloan, Department of Statistics, University of Manitoba, Winipeg. Manitoba, Canada R3T 2 N2.

\section{Program Name: $\quad$ SBAYES (Tiemey)}

Function:

The system consists of S-functions to compute approximations of posterior means, varinaces and marginal densities that are generally more accurate than the Lindley's Method mentioned above (see for reference: Tiemey and 


\author{
Kadane(1986)). \\ Programing language: FORTRAN 77 and $C$. Requires access to the $S$ package for implementation. \\ Availability: Available free of charge from Prof. Luke Tierney, School of Statistics, \\ University of Minnesota, Minneapolis, MN 55113.
}

\title{
5. Elicitation of Prior Information.
}

Program Name: BAYES (Schervish)

Function:

This program elicits priors and fikis posterior and predictive distruibutions for samples from normal or binomial data with natural conjugate priors or mixed conjugate plus point mass priors. It also handles flat priors over bounded regions for normal data.

Programing language: FORTRAN IV, requires access to IMSL.

Machine:

DEC-2060. Graphics are good for GIGI teminals only.

Availability: The program is not yet ready for distribution. Available on request from Prof. Mark Schervish, Department of Statistics, Carnegie Mellon University, Pittsburgh, PA 15213.

Program Name: [B/D] [ Beliefs adjusted by Data (Goldstein/Wooff)]

Function:

The program, in final development stage, provides an interactive, interpretive subjectivist analysis of general (partialy specified, exchangeable) beliefs as described in Goldstein(1987a,b,c).

Output: $\quad$ The program output provides summaries of as to how and why beliefs are (i) expected to change and (ii) actually change as well as system diagnostics based on comparison of (i) and (ii).

Programing language: PASCAL

Availability:

will be available at cost of mailing and manual production from Prof. Michael Goldstein, Department of Statistics, University of Hull, Cottingham Road, Hull, U.K.

6. Reliability Analysis.

Program Name: BASS[ Bayesian Analysis for Series Systems(Martz)]

Function:

This program performs a Bayesian reliability analysis of series systems of independent binomial subsystems and components for either prior or test data at the component, subsystem and overall system level. It uses a beta prior for 
the survival probabilities.

Programing language: FORTRAN 77

Machines:

Portable, but requires DISPLA software package for graphics.

Availability:

Free of charge from Dr. Harry F. Martz, Group S-1, MS F600, Los Alamos

National Laboratory, Los Alamos, NM 87545.

Program Name: BURD [ Bayesian Updating of Reliability Data (Martz)]

Function:

Documentation: $\quad$ Ahmed,S., Metcalf, D. R., Clark, R. E. and Jacobsen J. A. (1981) BURD-

A Computer program for Bayesian updating of reliability data, NPGD-TM-582, Babcox and Wilcox Inc., Lynchburg, VA.

Program Name: IPRA[An Interactive PC-Based Procedure for Reliability Assessment (Singpurwalla)]

Function: $\quad$ A menu driven program performs a prioi assessment based on expert opinion or informed judgement for Weibull distributed life length data and the posterior analysis in a highly interactive manner, Singpurwalla(1986a). It also allows the incorporation of the analyst's opinion on the expertise of the experts.

Input: On-line data entry or use of menu option to store data in a file for a later use in the analysis.

Output: $\quad$ The program computes the marginal and joint posterior densities of the Wiebull parameters. The prior and posterior reliability functions for a specified time interval as well as distributions of reliability for specifed mission times can be computed. These quantities can be displayed in a tabular or 2-d/3-d graphics form or saved on disk.

Programing language: IBM BASIC

Machines: IBM PC or compatibles with math co-processor and graphics board. Documentation: Aboura, K. N. and Soyer, R.(1986) 'A User's msnial for an Interactive PC-Based Procedure for Reliability Assesment, Tech. Repont GWU/IRRA/ 


\begin{abstract}
Availability: The program diskette and user's manual are available from Prof. Nozer Singpurwalla, Department of Operations Research, George Washington University, Washington, D.C. 20052 at a nominal charge.
\end{abstract}

Program Name: IPNDIAn Interactive PC-Based System for Predicting the number of defects due to fatigue in Railroad Tracks(Singpurwalla)]

Function:

Input:

Output:

Programing language:

Machines:

Documentation:

Availability:

Remarks:
A menu driven program performs a Bayesian analysis of a non-homogeneous Poisson process with a Weibull intensity function in which the assessment of the prior information about the parameters is induced via an engineering model based on S-N curves, Singpurwalla(1986b). The procedure is applied to the prediction of the number of defects due to fatigue in railroad tracks.

On-line data entry or use of menu option to store data in a file for a later use in the analysis.

The program computes the marginal and joint posterior densities of the parameters in the Wiebull intensity function. The prior and posterior distribution of the number of defects due to fatigue over a time period is also computed. These quantities can be displayed in a tabular or $2-d / 3-d$ graphics form or saved on disk.

IBM BASIC

IBM PC or compatibles with math co-processor and graphics board. Choksy, M. and Daryanani, S.(1987) 'An interactive PC-Based System for Predicting the Number of Defects due to Fatigue in Railroad Tracks: User's Manual" Tech. Report GWU/IRRA/ Serial TR-87-3, George Washington University, Washington, D.C.

The program diskette and user's manual are available from Prof. Nozer Singpurwalla, Department of Operations Research, George Washington University, Washington, D.C. 20052 at a nominal charge.

(i) Prof. Singpurwalla has communicated to us that this procedure and the program has been adopted by The Association of American Railrcads for the analysis of fatigue defects data in railroad tracks. This is an indication that availability of appropraite software would lead to a widespread use of Bayesian methodology. 
Program Name: PREDSIM[Prediction and Simulation for mixtures of exponentials(Sloan)] Function: This program performs a Monte Carlo simulation of sampling from a mixtures of exponentials model using a method proposed by Marsaglia and computes Bayes estimates of the systematic parameters and reliability function and predictive intervals for future observations.

Programing language;

PL/I

Machine:

Portable but requires access to IMSL.

Availability:

Available free of charge from Prof. Jeff A. Sloan, Department of Statistics, University of Manitoba, Winipeg, Manitoba, Canada R3T 2N2.

\section{Miscelleneous.}

Program Name:

DISCBDIF(Stroud)

Function:

This $S A S^{\circledR}$ program classifies an input record into one of the two normal populations, based on training samples from each one. It uses either Geisser's discrimination procedure or a semi-diffuse limit of conjugate priors.

Programing language: A vailability: Requires access to SAS ${ }^{\circledR}$ packageand SAS ${ }^{\otimes}$ PROC MATRIX. available free of charge from Prof. Thomas W.F.Stroud, Department of Mathematics and Statistics, Queen's University, Kingston, Ontario K7L3N6.

\section{Concluding Remarks.}

The CADA monitor was the first general purpose program for Bayesian data analysis. It has gone through several enhancements and at this time is the only general purpose package. Even though CADA was presented at several SBIE seminars and it was available in various machine versions, we believe that it has not been accepted as 'the package' for Bayesian data analysis. This is mainly because all the analyses in CADA are carried out under a noninformative or a simplistic conjugate prior framework and it has had no facility for numerical integration. Thus it precludes Bayesian analysis for some realistic prior specifications. Furthermore, the BASIC language does not provide today's state of the art computing environment. The graphical interfaces in CADA have been almost non-existent. The package was probably installed at almost all US universities with Bayesian statisticians, but was not used extensively for teaching courses. Thus the circle of CADA awareness has been quite limited. There didn't seem to be any strong interest among the participants of the Bayesian Computing Workshop last year, to go with CADA as the base for the future development of a suitable Bayesian Package. 
The existing enhanced version of the CADA monitor seems to be quite obsolete to us as it has not changed the basic computing environment from the earlier version. On the other hand, the package is now being marketed by a private company and depending on their future development strategy, the algorithms in CADA could become a vehicle for a state of the art system. We need to explore the future plans of the CADA group before deciding about our own strategy.

The implementation of the Bayesian paradigm for a realistic data analysis would require a variety of numerical integration and approximation routines. The growth of the methodo'ogy and software for this has been phenomenal. But we still have a long way to go for approximation and numerical integration procedures and graphical displays for high dimensional problems.

Several researchers are in the process of developing general purpose software for Bayesian analysis of data from various sampling distributions and models. We must mention the software for Bayesian dynamic linear modeling and forecasting, see, West, Harrison and Migon(1985) and West and Harrison(1986), which will be an excellent addition to the Bayesian arsenal.

The strategy of writing all Bayesian software in $\mathrm{S}$ compatible routines sounds appealing from the point of view of researchers in Statistics deparments, where UNIX is slowly becoming a defacto operating system. However, $S$ in not accesssible to a large group of statisticians and other researchers in Business schools, and Economics and Engineering departments. On the other hand, it is wise to develop all new Bayesian software so that it could be incorporated in an already existing and widely acceptable computing environment. Thus one does not have to worrry about developing its data management and graphics capabilities and the students and data analysts do no: have to leam yet another system. The only way to develop a quickly acceptable Interactive Bayesian Software Package is to adopt some of the existing main programs and subroutines as modules in some widely used statistics package which is available for mini and micro computers and add more and more modules to it as the new methodology and its software are developed. It is about time that all of us agree on one option. We believe that the most suitable package for this purpose is MINITAB, since it is very widely used for teaching and data analysis for moderate sized data sets. Furhermore, with a proper approach to Minitab Inc., we could receive suitable cooperation from them.

\section{References}

Carlin, J.B., Dempster, A. and Jonas, J.B.(1985) 'Bayesian estimation of unobserved components in time series', Jour. of Econometrics $3067-90$.

Caves, D.W.,Herriges, J.A., Train, K.A. and Windle, R.J.(1987) 'A Bayesian approach to 
combining conditional demand and engineering models of electricity usage' Review of Economics and Statistics, To appear.

Geweke, John(1987) 'Bayesian inference in econometric models using Monte Carlo integration', DP:87-2, Institute of Statistics and Decision Sciences, Duke University.

Goldstein, M.(1987a) 'Systemic analysis of limited belief specifications', The Statistician, To appear Goldstein, M.(1987b) 'Can we build a subjectivist statistical package?', in Proc. Symposium in memorium of Bruno de Finetii, To appear.

Goldstein, M.(1987c) 'The data trajectory', Presented at the Third Valencia International Meeting on Bayesian Statistics, Altea, Spain

Jaynes, E.T.(1983) 'Prior information and ambiguity in inverse problems' Proc. AMS-SIAM Symposium on Inverse Problems, New York.

Lindley, D.V.(1980) 'Approximate Bayesian methods', in Bayesian Statistics (Eds: J.M. Bernardo, M.H.DeGroot, D.V.Lindley and A.F.M.Smith), Valencia, Spain: University Press.

Monahan, John F.(1983) 'Fully Bayesian analysis of ARMA time Series models', Jour. of Econometrics $31307-331$.

Nebebe, F. and Stroud, T.W.F.(1986) 'Bayes and empirical Bayes estimation of regression coefficients', Canadian Jour. of Statist . 14 267-280.

Press, S. James(1980) 'Bayesian computer programs' in Bayesian Analysis in Econometrics and Statistics (ed. A. Zellner), Amsterdam: North Holland.

Racine, A., Grieve, A.P., Fluhler, H., and Smith, A.F.M.(1986) 'Bayesian methods in practice: Experiences in pharmaceutical industry (with discussion), Applied Statistics 35 93-150.

Singpurwalla, Nozer D.(1986a) 'An interactive PC-Based procedure for reliability assessment incorporating expert opinion and survival data' GWU/IRRA/Serial TR-86/1, Institute of Reliability and Risk Analysis, The George Washington University.

Singpurwalla, Nozer D.(1986b) 'An interactive PC-Based system for predicting the number of defects due to fatigue in railroad tracks' GWU/IRRA/Serial TR-86/7, Institute of Reliability and Risk Analysis, The George Washington University.

Smith. A.F.M., Skene, A.M., Shaw, J.E.H., Naylor, J.C., and Dransfield, M.(1985) 'The implementation of the Bayesian paradigm'Comm. Statist., Theo.\& Meth. 14(5) 1079-1102. Stewart, L.(1979) 'Multiparameter univariate Bayesian analysis'Jour. American Statist. Assoc., 74 684-693.

Stewart, L.(1983) ' Bayesian analysis using Monte Carlo integration and a powerful methodology for handling some difficult problems', The Statistician, 32 195-20u.

Stewart, L.(1985) 'Multiparameter Bayesian inference using Monte Carlo integration- Some 
techniques for bivariate analysis', in Bayesian Statistics 2 (Eds: J.M. Bernardo, M.H.DeGroot, D.V.Lindley and A.F.M.Smith), Amsterdam: North Holland

Thompson, P. and Miller, R. B.(1986) 'Sampling the future: A Bayesian approach to forecasting from univariate time series models', Jour. of Business and Économic Statistt., 4 427-436.

Tierney, L. and Kadane, J. (1986) 'Accurate approximations for posterior moirients and marginal densities', Jour. American Statist. Assoc., 81 82-86

West, M. and Harrison, P.J.(1986) 'Monitoring and adaptation in Bayesian forecasting models' Jour. American Statist. Assoc., 81 741-750.

West, M., Harrison, P.J., and Migon, H.S.(1985) 'Dynamic generalized linear models and Bayesian forecasting' (with discussion), Jour. American Statist. Assoc., 80 73-97. van Dijk, H.K., Kloek, T., and Boender, C.G.E.(1985) 'Posterior moments computed by mixed integrtion', Jour. of Econometrics , 29 3-18. 